\title{
Does Functional Connectivity Provide a Marker for Cognitive Rehabilitation Effects in Alzheimer's Disease? An Interventional Study
}

\author{
Sina Ochmann ${ }^{\mathrm{a}, *}$, Martin Dyrba ${ }^{\mathrm{a}}$, Michel J. Grothe ${ }^{\mathrm{a}}$, Elisabeth Kasper ${ }^{\mathrm{a}}$, Steffi Webel $^{\mathrm{a}}$, \\ Karlheinz Hauenstein ${ }^{\mathrm{c}}$ and Stefan J. Teipel ${ }^{\mathrm{a}, \mathrm{b}}$ \\ ${ }^{a}$ DZNE, German Center for Neurodegenerative Diseases, Site Rostock/Greifswald, Germany \\ ${ }^{\mathrm{b}}$ Department of Psychosomatic Medicine, University of Rostock, Rostock, Germany \\ ${ }^{\mathrm{c}}$ Institute of Diagnostic and Interventional Radiology, University Medicine Rostock, Rostock, Germany
}

Accepted 28 October 2016

\begin{abstract}
.
Background: Cognitive rehabilitation (CR) is a cognitive intervention for patients with Alzheimer's disease (AD) that aims to maintain everyday competences. The analysis of functional connectivity (FC) in resting-state functional MRI has been used to investigate the effects of cognitive interventions.

Objectives: We evaluated the effect of CR on the default mode network FC in a group of patients with mild AD, compared to an active control group.

Methods: We performed a three-month interventional study including 16 patients with a diagnosis of AD. The intervention group (IG) consisted of eight patients, performing twelve sessions of CR. The active control group (CG) performed a standardized cognitive training. We used a seed region placed in the posterior cingulate cortex (PCC) for FC analysis, comparing scans acquired before and after the intervention. Effects were thresholded at a significance of $p<0.001$ (uncorrected) and a minimal cluster size of 50 voxels.

Results: The interaction of group by time showed a higher increase of PCC connectivity in IG compared to CG in the bilateral cerebellar cortex. CG revealed widespread, smaller clusters of higher FC increase compared with IG. Across all participants, an increase in quality of life was associated with connectivity increase over time in the bilateral precuneus.

Conclusions: CR showed an effect on the FC of the DMN in the IG. These effects need further study in larger samples to confirm if FC analysis may suit as a surrogate marker for the effect of cognitive interventions in AD.
\end{abstract}

Keywords: Alzheimer's disease, cognitive rehabilitation, default mode network, dementia, functional connectivity, functional MRI

\section{INTRODUCTION}

Cognitive interventions may be useful to reduce rates of cognitive decline in people with Alzheimer's

\footnotetext{
${ }^{*}$ Correspondence to: Sina Ochmann, DZNE German Center for Neurodegenerative Diseases, Site Rostock/ Greifswald, Gehlsheimer Straße 20, 18147 Rostock, Germany. Tel.: +49 381 494 9471; Fax: +49 381494 9472; E-mail: sina.ochmann@unirostock.de.
}

disease (AD) dementia or at risk for AD [1]. Cognitive rehabilitation $(\mathrm{CR})$ is a multimodal cognitive intervention that aims to preserve a patient's everyday activities, independent living, and quality of life [2]. CR combines the dimensions of individuality (e.g., pursuing personal goals with relevance to the patient's everyday life), compensation (e.g., maintaining skills and compensating deficits), integration (e.g., applying multimodal methods), and interaction 
(including the patient's environment) [3]. Several studies integrating key concepts of CR have shown an improvement of quality of life and a decrease in depressive symptoms in people with dementia, but no significant effects on everyday competence (activities of daily living, ADL) or cognitive functions [3-6].

To develop effective therapies, it is essential to better understand the effects of an intervention on underlying neuronal dysfunction in dementia. Functional connectivity networks comprise a dynamic synchronized activity between functionally coupled brain regions [7]. Researchers have employed imaging techniques [8-10] to study progressive disruption of functional connectivity networks as a possible mechanism underlying the clinical course of $\mathrm{AD}$ [11-13]. The most widely studied network so far is the default mode network (DMN), which is characterized by a task-related deactivation in functional magnetic resonance imaging (fMRI). Typically, it shows highest activity in a wakeful resting-state in the precuneus/posterior cingulate cortex (PCC), medial prefrontal cortex (MPFC), lateral temporal and parietal cortex, thalamus, and hippocampus [14]. Resting-state fMRI (rs-fMRI) [15] allows the study of patients who are not able to follow complex activation paradigms during fMRI, and the DMN can be readily reconstructed from rs-fMRI by seed-based connectivity analysis of its principle network nodes, most typically the PCC $[16,17]$. The progressive decrease of functional connectivity in the DMN facilitates the differentiation between healthy controls, mild cognitive impairment (MCI), and AD dementia [13, 18, 19] but may also provide a marker to track the disease's progress. So far, only a few studies have investigated longitudinal changes in the DMN in patients with MCI and AD. Studies indicate a fractionation of the DMN in several subsystems which disintegrate consecutively [20-22]. Longitudinal studies also suggested an increase of connectivity between the PCC and frontal regions in early stages of AD [20, 23], and an increase of connectivity between PCC and precuneus in a group of MCI patients having converted to AD compared to non-converters [24]. These connectivity increases have typically been interpreted in the context of compensatory mechanisms. Even fewer studies have investigated effects of cognitive interventions on rs-fMRI connectivity. Cognitive training in healthy older people led to initial small increases in functional connectivity between the hippocampus and superior temporal gyrus regions after three weeks, which were lost after the complete training of 12 weeks [25]. In people with MCI an eight weeks meditation-intervention resulted in increased connectivity between PCC and MPFC/hippocampus without changes in behavioral parameters [26]. Functional imaging techniques other than rs-fMRI revealed effects of CR in AD and MCI. Using a recognition-task fMRI paradigm, Van Paasschen found that eight weeks of CR in patients with early-stage $\mathrm{AD}$ resulted in an increase of activation in the intervention compared to the control group in frontal and parietal regions, but had no significant effects on behavioral performances. The authors suggested that fMRI may be more sensitive to intervention effects than neuropsychological tests [27]. In an $\left[{ }^{18} \mathrm{~F}\right]$ fluorodeoxyglucose (FDG) positron emission tomography (PET) study, six months CR in patients with MCI and AD led to attenuated metabolic decline in both intervention groups compared to the active control group, but the authors found only nonsignificant correlations between FDG-uptake and cognitive parameters [28]. In summary, functional imaging provides promising endpoints for CR interventions that deserve further study.

The aim of our study was to assess the effect of 12 weeks CR on functional connectivity within the $\mathrm{DMN}$ in patients with mild AD compared to an active control group.

We hypothesized that $\mathrm{CR}$, which is thought to mobilize cognitive resources, may alter functional connectivity in the DMN.

\section{METHODS}

\section{Subjects}

We screened 44 patients in the memory clinic of the University Medical Center Rostock and included 20 subjects with a diagnosis of clinically probable $\mathrm{AD}$ or possible $\mathrm{AD}$ with concomitant cerebrovascular disease (mixed dementia), according to NINCDSADRDA criteria [29]. Cerebrovascular comorbidity was diagnosed according to ICD 10 criteria [30]. Subjects who were taking psychotropic drugs needed to be under stable doses for at least three months. Almost all subjects were taking antidementive medication. In the control group, three subjects took donepezil, two subjects took rivastigmine, and three subjects did not take any antidementive medication. In the intervention group, three subjects took rivastigmine, four subjects took donepezil, and one subject took memantine. All patients received a comprehensive neurological and psychiatric examination. 
Table 1

Demographical measures at baseline

\begin{tabular}{|c|c|c|c|c|c|c|c|}
\hline \multirow[t]{2}{*}{ Parameters } & \multicolumn{3}{|c|}{$\begin{array}{c}\text { Intervention } \\
\text { group (IG) } n=8\end{array}$} & \multicolumn{3}{|c|}{$\begin{array}{l}\text { Control group } \\
\text { (CG) } n=8\end{array}$} & \multirow[b]{2}{*}{$p^{*}$} \\
\hline & Mean & SD & Range & Mean & SD & Range & \\
\hline Age (years)* & 70.4 & 8.72 & $59-83$ & 69.8 & 8.81 & $53-80$ & 0.916 \\
\hline Sex $(\text { male/female })^{+}$ & $4 / 4$ & & & $5 / 3$ & & & 0.614 \\
\hline Education (years)* & 14.4 & 2.26 & $11-17$ & 14.4 & 2.33 & $10-17$ & 0.957 \\
\hline MMSE (points)* & 21.8 & 3.24 & $17-25$ & 24 & 3.55 & $19-29$ & 0.297 \\
\hline
\end{tabular}

* Measured by U-test; ${ }^{+}$measured by chi-square-test; SD, standard deviation; MMSE, Mini-Mental Status Examination.

Exclusion criteria consisted of a history of neurological or psychiatric illness (other than AD), or extensive pathological changes in the MRI scan, such as a tumor or evidence of stroke. The study was approved by the ethics committee of the University Medical Center Rostock and registered in the database of clinicaltrials.gov (A 2014-0113). All subjects and their representatives provided written informed consent. The study was conducted in compliance with the Declaration of Helsinki of 1964, last amended by 64th WMA General Assembly, Fortaleza, Brazil, October 2013.

\section{Study design}

Our study examined the effects of a three-month cognitive rehabilitation program in a semi-controlled design. Details on the design are described in detail in our companion paper [31]. After screening, 20 patients were partially randomized to either an intervention group (IG) or control group (CG). We conducted an adaptive and partially balanced randomization. As five subjects originated from a pilot trial waiting group and were already predetermined for the intervention group, we were forced to conduct an adaptive randomization at baseline. The remaining subjects $(n=15)$ passed a computer-based balanced randomization. At baseline, the two groups did not differ significantly with respect to age, sex, education, and MMSE (Table 1). Moreover, they did not differ with respect to severity of AD according to MMSE (Table 1) and CERAD-scores (here not reported in detail). Eight subjects per group completed the study. For details on design and dropouts, please see Fig. 1. The intervention group received a cognitive rehabilitation intervention based on the CORDIAL-program [32], as described in detail in our companion paper [31]. In short, this program includes 6 modules in 12 weekly sessions over three months with contents such as identifying

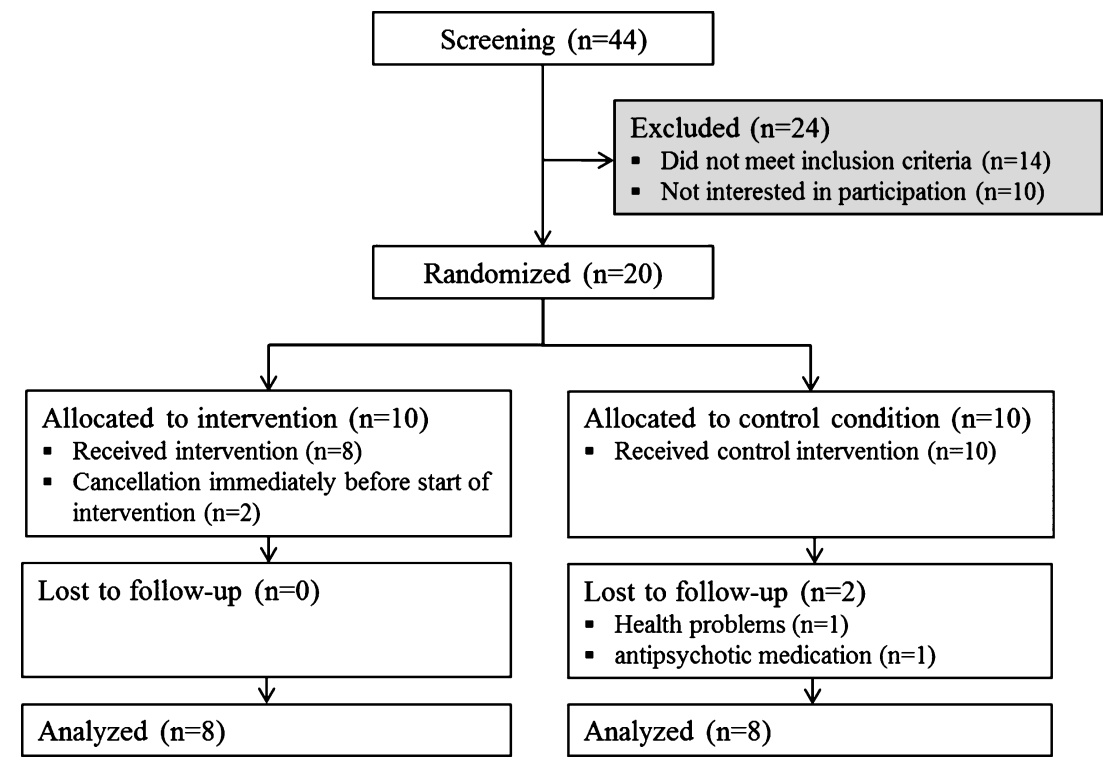

Fig. 1. Flowchart of randomization and procedure according to CONSORT criteria [58]. 
individual problems, defining personal goals, biographical work, implementation of pleasant activities and external memory aids, concluded by an evaluation session with individual plans for the future. The control group received standardized cognitive training [33] with worksheets for homework and monthly meetings.

\section{Neuropsychological outcome}

As primary outcome, we used two scales, measuring Activities of daily living (ADL-competence). Firstly, we used the Bayer's Activities of Daily Living Scale: Bayer-ADL [34], addressing everyday competence. Secondly, we employed the Nuremberg Aging Observation Scale (NSL) [35], completed by both the patients (NSL-P) and the caregivers (NSL-C). Secondary outcomes were the cognitive status, measured with the Consortium to establish a registry for $\mathrm{AD}$ (CERAD) [36], and non-cognitive domains, for example depression, measured with the Geriatric Depression Scale (GDS) [37], and quality of life, measured by the Health-related quality of life for people with dementia (DEMQOL) [38]. The neuropsychological testing was conducted blinded to the participants' intervention condition.

\section{Image data acquisition}

We used a 3T Siemens Magnetom VERIO scanner (Erlangen, Germany) with a 32-channel head coil. The patients were instructed to lie still, keep their eyes closed and not to fall asleep. The field-of-view (FOV) spanned the whole brain and was aligned along the anterior-to-posterior commissure line. The functional MRI was based on echo-planar imaging using a $64 \times 64$ image matrix with 47 axial slices (spacing $3.5 \mathrm{~mm}$, thickness $3.5 \mathrm{~mm}$, no gap, interleaved acquisition). The FOV was $224 \times 224 \times 165 \mathrm{~mm}$, voxel size $3.5 \times 3.5 \times 3.5 \mathrm{~mm}^{3}$, echo time $30 \mathrm{~ms}$, repetition time $2,580 \mathrm{~ms}$, flip angle $80^{\circ}$, and parallel imaging acceleration factor 2 . We obtained 180 volumes within $7 \mathrm{~min} 54 \mathrm{~s}$. During the same session, a high-resolution T1-weighted anatomical image was acquired, applying the magnetization-prepared rapid gradient echo (MPRAGE) sequence with the following parameters: $256 \times 256$ image matrix with 192 sagittal slices, FOV $256 \times 256 \times 192 \mathrm{~mm}$, voxel size $1 \times 1 \times 1 \mathrm{~mm}^{3}$, echo time $4.82 \mathrm{~ms}$, repetition time $2,500 \mathrm{~ms}$, flip angle $7^{\circ}$ and parallel imaging acceleration factor 2. The duration of the sequence was $9 \min 20 \mathrm{~s}$.

\section{Image processing}

All functional and anatomical scans were visually inspected to ensure data quality. We used SPM8 (Wellcome Trust Centre for Neuroimaging) implemented in MATLAB R2103a (The MathWorks, Inc.) in conjunction with the Voxel-Based Morphometry toolbox (VBM8, r413 [39]) and the Data Processing Assistant for Resting-State fMRI (DPARSF advanced edition, Version 3.1 [40]) for data processing. The anatomical $\mathrm{T}_{1}$-weighted scans were processed following the longitudinal pipeline of the VBM8 toolbox. Bias-corrected mean images were calculated from the realigned anatomical images of the baseline (BL) and follow-up (FU) time points. The anatomical images were segmented into grey matter, white matter, and cerebrospinal fluid partitions using VBM8. The Diffeomorphic Anatomical Registration Through Exponentiated Lie algebra (DARTEL) algorithm [41] was used to create a standardized template and to normalize the $\mathrm{T}_{1}$-weighted images to the Montreal Neurological Institute (MNI) reference coordinate system [42]. We removed the first six volumes from the functional images to ensure scanner gradient field stabilization; the data underwent slice-time correction and were realigned to the mean functional image of the time series. Then, we co-registered the fMRI images to the anatomical $\mathrm{T}_{1}$-weighted mean image using the temporal mean as reference. We regressed out nuisance signals due to head motion (6 movement parameters + first-order derivatives), as well as the mean time courses for the global signal, white matter signal, and cerebrospinal fluid signal derived from the anatomically segmented maps. We applied temporal band-pass filtering in the frequency range 0.01 to $0.1 \mathrm{~Hz}$. For spatial normalization, we applied the deformation fields, obtained from the anatomical data, to transform the functional data to MNI standard space. As a final step, we smoothed the warped functional images with an $8 \mathrm{~mm}$ full-width-at-half-maximum (FWHM) Gaussian kernel.

\section{Statistical analysis}

We followed a seed-based approach to evaluate the functional connectivity of the DMN. As implemented in the DPARSF advanced edition toolbox (State Key Laboratory of Cognitive Neuroscience and Learning, Beijing, http://www.rfmri.org [40]), we calculated whole brain correlation maps of the signal time course 


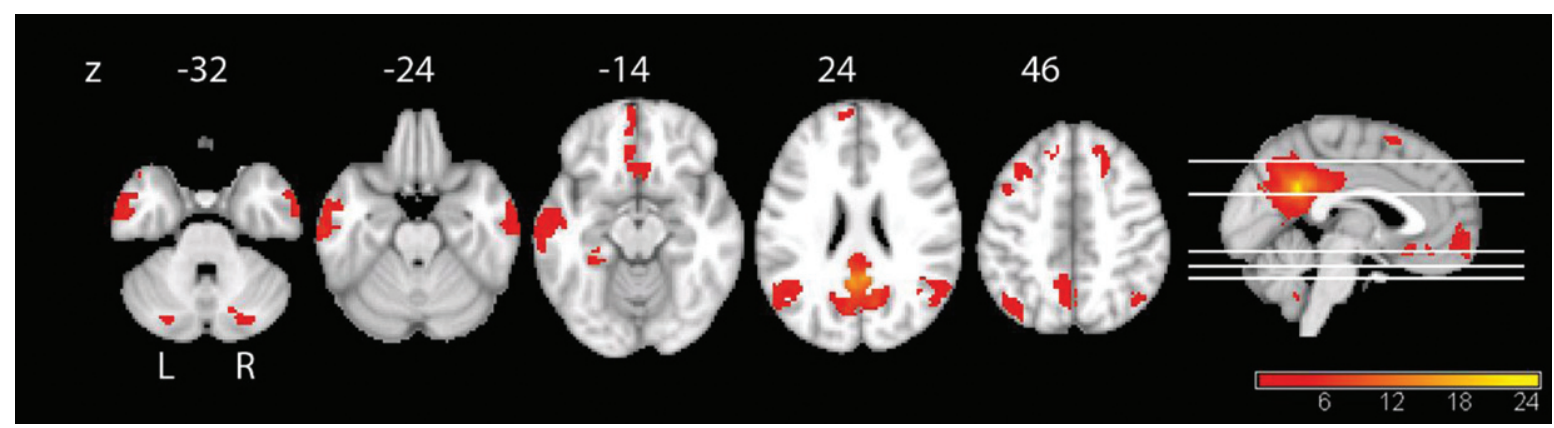

Fig. 2. Functional connectivity of the PCC in the full sample at baseline. The connectivity maps reveal the typical pattern of the DMN. We applied threshold of $p<0.05$, FWE-corrected with a minimal cluster size of 50.

using a seed sphere placed in the PCC at MNIcoordinates $(0,-51,29)$ with a radius of $6 \mathrm{~mm}[16,17$, 43]. In the same step, we applied the Fisher transform to calculate $\mathrm{z}$-score maps from the Pearson correlation coefficients. Using these maps, we conducted the following analyses in SPM8 (Wellcome Trust Centre for Neuroimaging, http://www.fil.ion.ucl.ac.uk):

First, we performed a one-sample $t$-test to determine the functional connectivity pattern within the whole sample $(p<0.05$ corrected for family-wise error, minimal cluster size of 50 voxels). Second, we performed a two-sample $t$-test to compare the groups at baseline and at follow-up. Third, we conducted a two-way repeated-measures ANOVA, using the flexible factorial design in SPM, on the main effect of group and condition, i.e., time between BL and FU, and the interaction effect for group and condition. Fourth, we performed a paired $t$-test to assess the main effect of time for each group separately. In addition, we calculated functional difference images, subtracting the BL-image from the FU-image, and assessed associations between the longitudinal differences in functional connectivity and the differences of a significant neuropsychological variable using voxel-wise linear regressions. All voxel-wise effects were assessed at a statistical threshold of $p<0.001$, uncorrected, and a minimal cluster size of 50 contiguous voxels. To identify the clusters' locations, we used the Automated Anatomical Labeling toolbox (2008) for SPM8 with a standard MNI-template.

\section{RESULTS}

From the initially included twenty patients, sixteen completed the study. Two subjects in each group dropped out due to various reasons (Fig. 1).

\section{Neuropsychological outcome}

There were no significant changes in the primary outcome of ADL-competence after intervention (Bayer-ADL: $\mathrm{F}=2.93, p=0.109$; NSL-Caregivers rating: $\mathrm{F}=0.02, \quad p=0.900 ; \quad$ NSL-patients-rating: $\mathrm{F}=1.11, p=0.310)$. Regarding the secondary outcome, we found a significant group $\times$ time interaction effect only for the quality of life index $(\mathrm{F}=8.15$, $p=0.013$ ), with an effect size of Cohen's $d=-1.43$. Further details regarding all neuropsychological outcomes can be found in our companion paper [31].

\section{Functional connectivity outcome}

The whole sample showed significant PCC functional connectivity in the precuneus, superior parietal cortex, lateral temporal cortex, and medial prefrontal cortex (Fig. 2), representing a typical DMN pattern [44]. A comparison of groups at baseline revealed for the IG, compared to the CG, higher connectivity in the right angular cortex and superior frontal cortex, whereas the CG showed higher connectivity in the left supplementary motor cortex. At followup, the IG revealed higher connectivity again in the right angular cortex and the CG showed higher connectivity than the IG in the left superior temporal cortex. Details are shown in Supplementary Table 1.

In the longitudinal analyses, the main effect of time showed an increase of PCC connectivity from $\mathrm{BL}$ to FU for the whole group in the left precentral gyrus, and to a lower extent in the precuneus bilaterally, the right PCC, and in the right inferior temporal lobe (Fig. 3). Connectivity decreased from BL to FU between the PCC and left temporal regions and the right middle temporal pole. Smaller clusters 

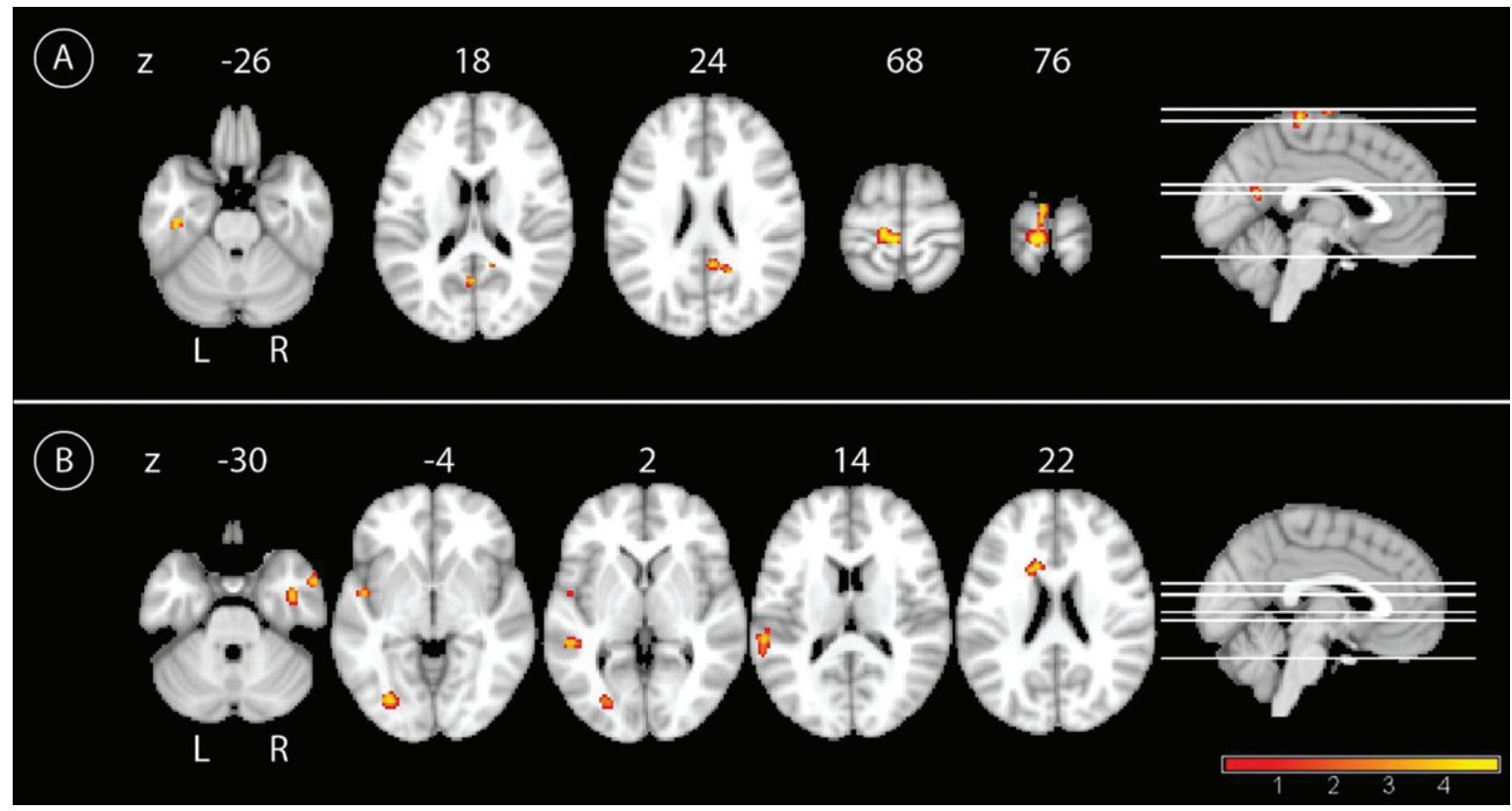

Fig. 3. Main effect of time in the whole sample. A) Results for the main effect of time with the contrast follow-up $>$ baseline in the whole sample. Yellow color represents higher connectivity with the PCC at FU compared to BL, $p<0.001$, uncorrected, and a minimal cluster size of 50. B) Results for the main effect of time with the contrast baseline $>$ follow-up in the whole sample. Yellow color represents higher connectivity with the PCC at BL compared to FU, $p<0.001$, uncorrected, and a minimal cluster size of 50.

of connectivity decrease were located in the left middle occipital cortex and the left caudate nucleus (Fig. 3 and Table 2). The interaction of group by time showed a higher increase of PCC connectivity in IG compared to CG over time primarily in the bilateral cerebellar cortex, with smaller clusters in the left postcentral gyrus, supplementary motor cortex, and orbital frontal cortex (Fig. 4). By contrast, CG showed a higher increase of PCC connectivity over time compared with IG in more widespread areas, including peaks in the left lingual cortex, temporal inferior lobe, orbito-frontal cortex, right precentral cortex, pallidum, and caudate nucleus (Fig. 4 and Table 2). The results of the paired $t$-test showed an increase of connectivity over time for IG in the following regions: left supplementary motor cortex, left postcentral gyrus, left precuneus, right insula, and left paracentral lobule. The opposite contrast showed a decrease of connectivity over time in IG in the left fusiform gyrus. In the CG, we found an increase of connectivity over time in the left temporal inferior lobe, right crus II of the cerebellum, and the right middle frontal lobe. Also for the CG, we found a decrease of connectivity over time in the regions left middle temporal lobe, left fusiform gyrus, and lobule VI of the right cerebellum (Table 3).
As quality of life revealed the only significant interaction effect in the behavioral analysis, we correlated this variable with the functional connectivity to reduce the number of comparisons. In the IG group, QoL increase was associated with connectivity increase over time in the bilateral precuneus, particularly on the left side, in the right middle temporal lobe, in the left gyrus calcarinus and in the right inferior parietal cortex (Fig. 5 and Table 4). In the CG group, QoL was not significantly associated with changes in connectivity.

\section{DISCUSSION}

Rs-fMRI has been proposed as a promising marker to track changes in functional connectivity throughout different stages of AD [20]. In our study, we assessed effects of CR on functional connectivity in patients with mild AD. Some studies reported small effects of cognitive interventions in functional imaging, such as rs-fMRI [25, 26], task fMRI [45], and FDG-PET [28]. These studies found no significant correlations between functional brain changes and cognitive endpoints. Studies varied in the type of intervention (meditation, cognitive training, CR), design aspects, i.e., duration of intervention (twelve 
Table 2

Longitudinal functional connectivity results

\begin{tabular}{|c|c|c|c|c|c|c|c|}
\hline \multirow[t]{2}{*}{ Contrast } & & \multirow[t]{2}{*}{$\begin{array}{l}\text { Brain Region, right } \\
\text { (R) or left (L) }\end{array}$} & \multirow{2}{*}{$\begin{array}{c}\text { Cluster } \\
\text { size } \\
\text { (voxel) }\end{array}$} & \multicolumn{3}{|c|}{$\begin{array}{c}\text { Peak MNI } \\
\text { coordinates }(\mathrm{mm})\end{array}$} & \multirow[t]{2}{*}{$\begin{array}{l}\text { peak-level } \\
\text { T-Statistic }\end{array}$} \\
\hline & & & & $\mathrm{x}$ & $\mathrm{y}$ & $\mathrm{z}$ & \\
\hline \multirow{11}{*}{$\begin{array}{l}\text { Longitudinal: } \\
\text { Main effect } \\
\text { of time }\end{array}$} & \multirow[t]{5}{*}{$\mathrm{FU}>\mathrm{BL}$} & Precuneus, R & 124 & 18 & -54 & 27 & 5.28 \\
\hline & & Precentral gyrus, L & 594 & -6 & -31 & 67 & 5.07 \\
\hline & & Temporal Inferior Lobe, L & 67 & -42 & -21 & -26 & 4.45 \\
\hline & & Precuneus, $\mathrm{R}$ & 51 & 12 & -48 & 13 & 4.17 \\
\hline & & Precuneus, L & 55 & -2 & -61 & 19 & 3.97 \\
\hline & \multirow[t]{6}{*}{$\mathrm{BL}>\mathrm{FU}$} & Middle Occipital Lobe, L & 175 & -28 & -76 & -2 & 5.61 \\
\hline & & Superior Temporal Lobe, L & 155 & -60 & -36 & 12 & 4.27 \\
\hline & & Temporal Inferior Lobe, $\mathrm{R}$ & 101 & 42 & -6 & -29 & 4.46 \\
\hline & & Corpus Nuclei Caudate, L & 96 & -16 & 14 & 24 & 4.83 \\
\hline & & Middle Temporal Lobe, L & 96 & -50 & -36 & 4 & 4.58 \\
\hline & & Middle Temporal Pole, $\mathrm{R}$ & 74 & 56 & 6 & -32 & 4.48 \\
\hline \multirow{12}{*}{$\begin{array}{l}\text { Longitudinal: } \\
\text { Interaction } \\
\text { effect }\end{array}$} & \multirow[t]{6}{*}{$\mathrm{IG}>\mathrm{CG}$} & Cerebellum, Lobule IV/V, R & 210 & 18 & -45 & -15 & 5.04 \\
\hline & & Cerebellum, Lobule VI, R & 175 & 18 & -55 & -33 & 4.82 \\
\hline & & Postcentral Cortex, L & 109 & -18 & -43 & 69 & 4.40 \\
\hline & & Inferior Frontal Cortex, Pars Orbitalis, R & 104 & 40 & 22 & -18 & 5.10 \\
\hline & & Supplementary Motor Cortex, L & 91 & 0 & -6 & 73 & 4.17 \\
\hline & & Cerebellum, Lobule III, R & 50 & 15 & -31 & -17 & 4.77 \\
\hline & \multirow[t]{6}{*}{$\mathrm{CG}>\mathrm{IG}$} & Lingual Cortex, L & 77 & -26 & -79 & -14 & 4.93 \\
\hline & & Inferior Temporal Lobe, $\mathrm{L}$ & 74 & -45 & -13 & -29 & 4.47 \\
\hline & & Precentral Cortex, $\mathrm{R}$ & 70 & 42 & -6 & 27 & 5.03 \\
\hline & & Nucleus Caudate, $\mathrm{R}$ & 65 & 20 & 15 & 24 & 4.06 \\
\hline & & Pallidum, R & 54 & 18 & -6 & -2 & 5.04 \\
\hline & & Orbitofrontal Cortex, L & 51 & -26 & 50 & -3 & 3.99 \\
\hline
\end{tabular}

Results of functional data from cross-sectional and longitudinal analysis, thresholded at $p<0.001$, uncorrected for multiple comparisons and a minimal cluster size of 50 voxel; IG- intervention group; CG, control group; MNI, Montreal Neurological Institute.
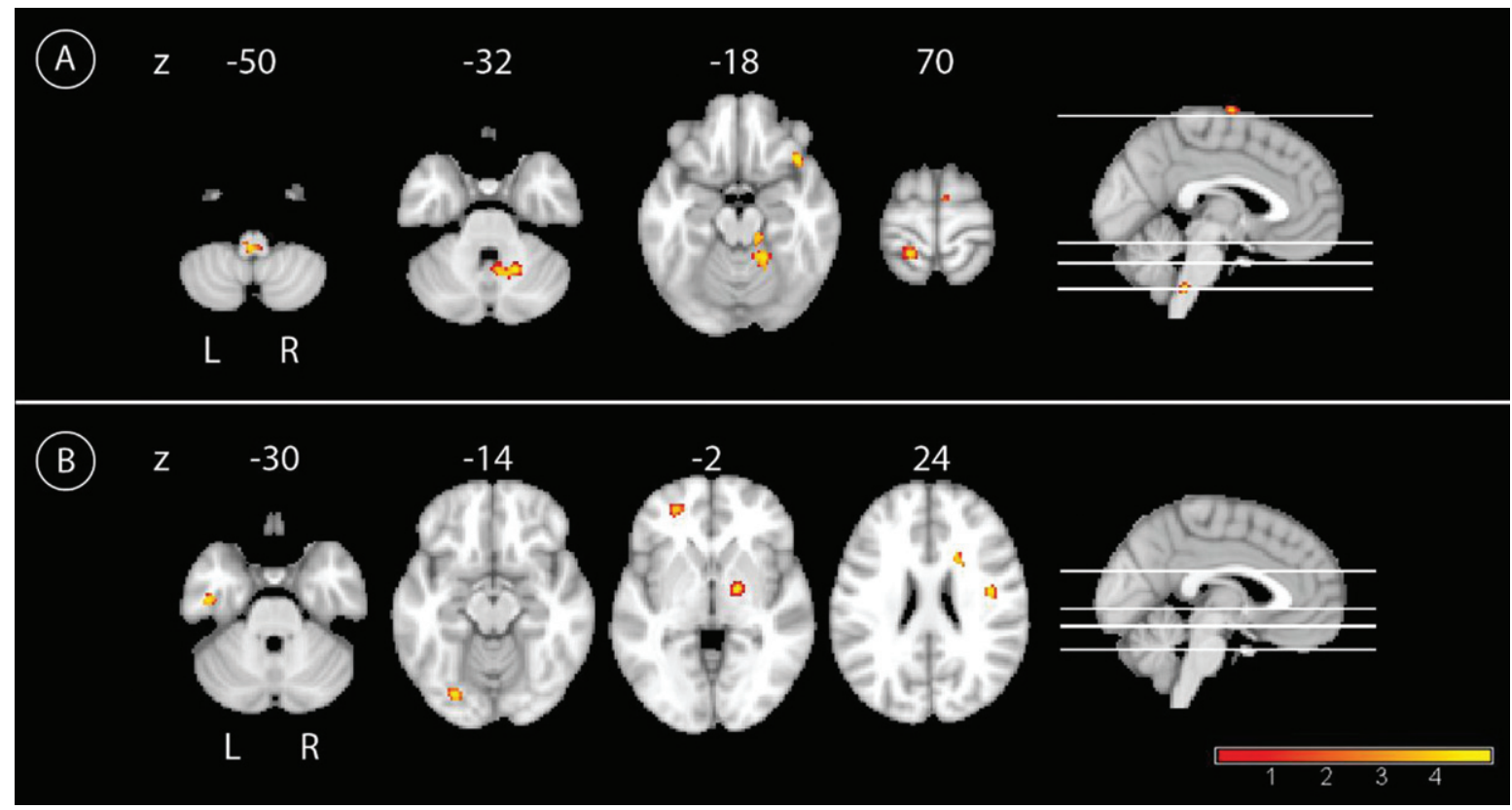

Fig. 4. Longitudinal interaction effect. A) Comparison of the longitudinal effect of the CR between groups with the contrast intervention group $>$ control group over time with the yellow color representing higher connectivity with the PCC in the IG compared to the CG, $p<0.001$, uncorrected, and a minimal cluster size of 50. B) The results for the interaction effect with the contrast control group $>$ intervention group with the yellow color representing higher connectivity with the PCC in the CG compared to the IG, $p<0.001$, uncorrected, and a minimal cluster size of 50 . 
Table 3

Longitudinal results for each group separately

\begin{tabular}{|c|c|c|c|c|c|c|c|}
\hline \multirow[t]{2}{*}{ Contrasts } & & \multirow[t]{2}{*}{$\begin{array}{l}\text { Brain Region, right } \\
\text { (R) or left (L) }\end{array}$} & \multirow{2}{*}{$\begin{array}{c}\text { Cluster } \\
\text { size } \\
\text { (voxel) } \\
\end{array}$} & \multicolumn{3}{|c|}{$\begin{array}{c}\text { Peak MNI } \\
\text { coordinates }(\mathrm{mm})\end{array}$} & \multirow[t]{2}{*}{$\begin{array}{l}\text { Peak-level } \\
\text { T-statistic }\end{array}$} \\
\hline & & & & $\mathrm{x}$ & $\mathrm{y}$ & $\mathrm{z}$ & \\
\hline \multirow[t]{6}{*}{ Longitudinal: IG } & $\mathrm{FU}>\mathrm{BL}$ & Supplementary Motor Cortex, L & 285 & 0 & -6 & 75 & 16.36 \\
\hline & & Postcentral Gyrus, L & 114 & -18 & -37 & 69 & 10.42 \\
\hline & & Precuneus, L & 86 & -12 & -27 & 64 & 9.19 \\
\hline & & Insula, R & 52 & 36 & 4 & -14 & 8.00 \\
\hline & & Paracentral Lobule, L & 58 & -12 & -52 & 64 & 7.99 \\
\hline & $\mathrm{BL}>\mathrm{FU}$ & Fusiform Gyrus, L & 119 & -21 & -72 & -21 & 6.16 \\
\hline \multirow[t]{6}{*}{ Longitudinal: CG } & $\mathrm{FU}>\mathrm{BL}$ & Temporal Inferior Lobe, L & 193 & -45 & -25 & -20 & 10.50 \\
\hline & & Cerebelum, Crus II, R & 122 & 50 & -54 & -45 & 9.30 \\
\hline & & Middle Frontal Lobe, R & 76 & 26 & 18 & 49 & 6.72 \\
\hline & $\mathrm{BL}>\mathrm{FU}$ & Middle Temporal Lobe, L & 88 & -50 & -34 & 3 & 11.64 \\
\hline & & Fusiform Gyrus, L & 97 & -27 & -76 & -5 & 9.47 \\
\hline & & Cerebelum, Lobule VI, R & 78 & 22 & -55 & -30 & 8.75 \\
\hline
\end{tabular}

Results from the paired $t$-Test comparing both timepoints for each group separately, thresholded at $p<0.001$, uncorrected for multiple comparisons and a minimal cluster size of 50 voxel. QoL, Quality of Life; IG, intervention group; CG, control group; MNI, Montreal Neurological Institute.

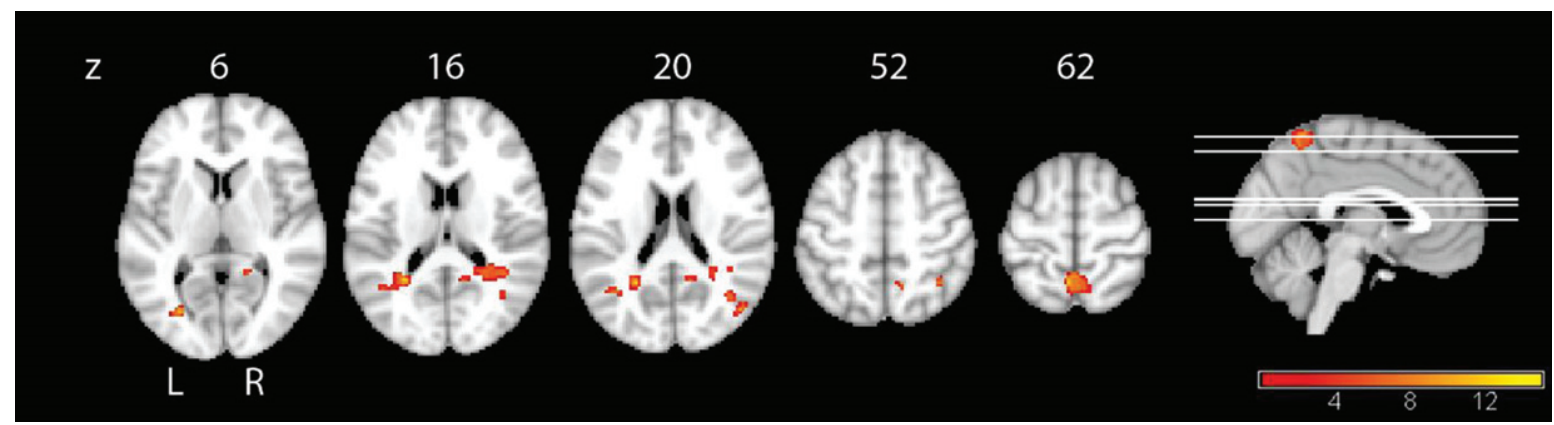

Fig. 5. Linear regression analysis of QoL and connectivity. Positive correlation of the QoL and functional difference images for the IG, with the yellow color representing regions that show increased connectivity with the PCC over time that are correlated with increased values in quality of life over time, $p<0.001$, uncorrected, and a minimal cluster size of 50 .

Table 4

Longitudinal results of QoL and functional connectivity correlation analysis

\begin{tabular}{|c|c|c|c|c|c|c|c|}
\hline \multirow[t]{2}{*}{ Contrasts } & & \multirow[t]{2}{*}{$\begin{array}{l}\text { Brain Region right } \\
\text { (R) or left (L) }\end{array}$} & \multirow[t]{2}{*}{$\begin{array}{c}\text { Cluster } \\
\text { size (voxel) }\end{array}$} & \multicolumn{3}{|c|}{$\begin{array}{c}\text { MNI peak } \\
\text { coordinates }(\mathrm{mm})\end{array}$} & \multirow[t]{2}{*}{$\begin{array}{l}\text { Peak-level } \\
\text { T-Statistic }\end{array}$} \\
\hline & & & & $\mathrm{x}$ & $\mathrm{y}$ & $\mathrm{z}$ & \\
\hline \multirow[t]{8}{*}{ QoL - IG } & Positive Correlations & Precuneus, L & 461 & -4 & -54 & 63 & 4.76 \\
\hline & & & 295 & -27 & -52 & 16 & 4.77 \\
\hline & & Precuneus, R & 104 & 22 & -48 & 27 & 4.00 \\
\hline & & Middle Temporal Lobe, $\mathrm{R}$ & 324 & 40 & -48 & 13 & 3.92 \\
\hline & & & 52 & 46 & -72 & 21 & 4.52 \\
\hline & & & 50 & 40 & -63 & 18 & 3.98 \\
\hline & & Gyrus Calcarinus, L & 82 & -27 & -73 & 6 & 4.53 \\
\hline & & Inferior Parietal Cortex, $\mathrm{R}$ & 65 & 38 & -54 & 52 & 4.30 \\
\hline
\end{tabular}

Linear regression with the functional difference-images as a dependent and the difference-values of the behavioral parameter QoL as an independent variable (assessed with the DEMQOL), thresholded at $p<0.001$, uncorrected for multiple comparisons and a minimal cluster size of 50 voxel. QoL, Quality of Life; IG, intervention group; CG, control group; MNI, Montreal Neurological Institute.

weeks, eight weeks, six months), and the target groups ( $\mathrm{AD}$, mild cognitive impairment, healthy elderly subjects) $[25,26,28,45]$.
At baseline, the full sample of our study showed the typical pattern of the DMN, replicating previous findings in similar cohorts $[13,18]$. Additionally, 
we compared both groups at baseline and found differences in a few regions, for instance higher connectivity in the right angular cortex in IG. However, this cluster also showed at follow-up, when comparing both groups, thus we do not expect an interference with other results. The interaction effect over time revealed an increase of connectivity with the PCC in regions of the cerebellum in the IG compared with the CG group. Studies have indicated that the cerebellum is relevant to all resting-state networks including the DMN $[46,47]$ and that the functional connectivity of the cerebellum also shows decline in AD [48, 49]. Furthermore, the cerebellum has been shown to take part in procedural or implicit memory [50, 51], which is known to be relatively preserved throughout the progress of $\mathrm{AD}$ compared to declarative memory [52]. Since our CR aimed at the implementation of routines, an effect on the functional connectivity between the PCC and the cerebellum may be plausible. CG showed increases of functional connectivity over time relative to the IG group in several brain regions, although with smaller clusters when compared to the contrast IG >CG. Still, the fMRI data did not provide unambiguous evidence for IG effects compared with CG. Similarly, a previous rs-fMRI study did not find clear effects of a cognitive intervention [25].

Irrespective of the treatment, connectivity with the PCC increased from baseline to follow-up in the bilateral precuneus and paracentral in the whole sample. We might interpret the increase of connectivity as an unspecific effect of intervention. On the one hand, we would expect that the cognitive intervention improved PCC function, as the region of precuneus and PCC represents a central hub within the DMN [53] with important contributions to the control of attention [21]. On the other hand, our results contradict the classical hypothesis of an early deterioration of this region in the disconnection cascade of the DMN from MCI to AD [20, 54], which would have expected a decrease over time. Possibly, due to the region's high connectivity, it is still capable to activate cognitive resources. Such compensating processes have been suggested to be part of the network's deterioration, as several researchers described an increase of connectivity between the $\mathrm{PCC} /$ precuneus region and frontal areas [20,23]. We also noticed a decrease of connectivity over time in the whole sample, especially in temporal regions. Generally, this aligns with the progression of neural degeneration in $\mathrm{AD}$, in which temporal regions are affected already at early stages [16, 55-57]. Analysis within the treatment groups suggest that these effects are mainly driven by the IG sample, but due to the small number of cases in either group, this interpretation requires independent confirmation.

When we assessed the effect of quality of life as the only neuropsychological endpoint that was significantly associated with IG versus CG intervention, we found an increase of quality of life to be associated with an increase of connectivity in the precuneus and the middle temporal lobe, but only in the IG. This was a post hoc comparison, where the independent variable was determined from the most significant behavioral effects. Therefore, we can only speculate on the meaning of such effect. There was a decrease of activity in the temporal lobe from BL to FU in the whole group. Thus the increase of quality of life, which only occurred in the IG group, might facilitate the compensating effects of cognitive rehabilitation on the temporal lobe.

In addition, one needs to consider the influence of medication on the outcome of behavioral and functional connectivity values, especially since the two groups differed slightly with regard to antidementive medication. We attempted to limit this effect, ensuring stable doses in each patient three months prior and during the intervention. Furthermore, we cannot rule out an effect of head motion, since the whole sample consisted of patients with AD. Nevertheless, maximum head motion correction revealed that none of the subjects exceeded $1.5 \mathrm{~mm}$. Only two subjects showed minimal head motion at baseline, one subject from the control group showed $1 \mathrm{~mm}$, and another subject from the control group $0.5 \mathrm{~mm}$. Thus, we expect only minimal interference from head motion.

The main limitation of our study constitutes the small sample size and therefore, all our results need replication in larger studies.

\section{Conclusions}

In conclusion, our results suggest that the analysis of functional connectivity provides valuable insights into the effects of CR on the brain that are worth to be further investigated, i.e., the role of the precuneus in compensatory processes and the function of the cerebellum within the DMN. It is important to further investigate why different concepts of treatment may have different effects on resting-state networks in order to better target vulnerable and compensatory cortical networks, to slow down progression of the disease and to enhance cognitive resources. 


\section{ACKNOWLEDGMENTS}

Stefan J. Teipel received support by a grant of the Federal Ministry of Research (BMBF) (AgeGain, 1GQ1425B).

Authors' disclosures available online (http://j-alz. com/manuscript-disclosures/16-0773r2).

\section{SUPPLEMENTARY MATERIAL}

The supplementary material is available in the electronic version of this article: http://dx.doi.org/ 10.3233/JAD-160773.

\section{REFERENCES}

[1] Rodakowski J, Saghafi E, Butters MA, Skidmore ER (2015) Non-pharmacological interventions for adults with mild cognitive impairment and early stage dementia: An updated scoping review. Mol Aspects Med 43-44, 38-53.

[2] Kinsella GJ, Mullaly E, Rand E, Ong B, Burton C, Price S, Phillips M, Storey E (2009) Early intervention for mild cognitive impairment: A randomised controlled trial. J Neurol Neurosurg Psychiatry 80, 730-736.

[3] Kasper E, Ochmann S, Hoffmann W, Schneider W, Cavedo E, Hampel H, Teipel S (2015) Cognitive rehabilitation in Alzheimer's disease - a conceptual and methodological review. J Prev Alzheimers Dis 2, 142-152.

[4] Buschert VC, Giegling I, Teipel SJ, Jolk S, Hampel H, Rujescu D, Buerger K (2012) Long-term observation of a multicomponent cognitive intervention in mild cognitive impairment. J Clin Psychiatry 73, 8.

[5] Viola LF, Nunes PV, Yassuda MS, Aprahamian I, Santos FS, Santos GD, Brum PS, Borges SM, Oliveira AM, Chaves GF, Ciasca EC, Ferreira RC, Paula VJ, Takeda OH, Mirandez RM, Watari R, Falcao DV, Cachioni M, Forlenza OV (2011) Effects of a multidisciplinar cognitive rehabilitation program for patients with mild Alzheimer's disease. Clinics 66, 1395-1400.

[6] Schiffczyk C, Romero B, Jonas C, Lahmeyer C, Muller F, Riepe MW (2013) Efficacy of short-term inpatient rehabilitation for dementia patients and caregivers: Prospective cohort study. Dement Geriatr Cogn Disord 35, 300-312.

[7] Fox MD, Raichle ME (2007) Spontaneous fluctuations in brain activity observed with functional magnetic resonance imaging. Nat Rev Neurosci 8, 700-711.

[8] Hendrix JA, Finger B, Weiner MW, Frisoni GB, Iwatsubo T, Rowe CC, Kim SY, Guinjoan SM, Sevlever G, Carrillo MC (2015) The Worldwide Alzheimer's Disease Neuroimaging Initiative: An update. Alzheimers Dement 11, 850-859.

[9] Teipel SJ, Meindl T, Grinberg L, Heinsen H, Hampel H (2008) Novel MRI techniques in the assessment of dementia. Eur J Nucl Med Mol Imaging 35(Supp 1), 69.

[10] Valkanova V, Ebmeier KP (2014) Neuroimaging in dementia. Maturitas 79, 202-208.

[11] Brier MR, Thomas JB, Ances BM (2014) Network dysfunction in Alzheimer's disease: Refining the disconnection hypothesis. Brain Connect 4, 299-311.

[12] Cabral J, Kringelbach ML, Deco G (2014) Exploring the network dynamics underlying brain activity during rest. Prog Neurobiol 114, 102-131.
[13] Dennis EL, Thompson PM (2014) Functional brain connectivity using fMRI in aging and Alzheimer's disease. Neuropsychol Rev 24, 49-62.

[14] Shulman GL, Fiez JA, Corbetta M, Buckner RL, Miezin FM, Raichle ME, Petersen SE (1997) Common blood flow changes across visual tasks: II. Decreases in cerebral cortex. J Cogn Neurosci 9, 648-663.

[15] Biswal B, Yetkin FZ, Haughton VM, Hyde JS (1995) Functional connectivity in the motor cortex of resting human brain using echo-planar MRI. Magn Reson Med 34, 537541.

[16] Greicius MD, Srivastava G, Reiss AL, Menon V (2004) Default-mode network activity distinguishes Alzheimer's disease from healthy aging: Evidence from functional MRI. Proc Natl Acad Sci U S A 101, 4637-4642.

[17] Brier MR, Thomas JB, Snyder AZ, Benzinger TL, Zhang D, Raichle ME, Holtzman DM, Morris JC, Ances BM (2012) Loss of intranetwork and internetwork resting state functional connections with Alzheimer's disease progression. J Neurosci 32, 8890-8899.

[18] Hafkemeijer A1, van der Grond J, Rombouts SA (2012) Imaging the default mode network in aging and dementia. Biochim Biophys Acta 1822, 431-441.

[19] Beason-Held LL (2011) Dementia and the default mode Curr Alzheimer Res 8, 361-365.

[20] Damoiseaux JS, Prater KE, Miller BL, Greicius MD (2012) Functional connectivity tracks clinical deterioration in Alzheimer's disease. Neurobiol Aging 33, 828.e19-30.

[21] Leech R, Kamourieh S, Beckmann CF, Sharp DJ (2011) Fractionating the default mode network: Distinct contributions of the ventral and dorsal posterior cingulate cortex to cognitive control. J Neurosci 31, 3217-3224

[22] Andrews-Hanna JR, Reidler JS, Sepulcre J, Poulin R, Buckner RL (2010) Functional-anatomic fractionation of the brain's default network. Neuron $\mathbf{6 5}, 550-562$.

[23] Wang Z, Liang P, Jia X, Jin G, Song H, Han Y, Lu J, Li $\mathrm{K}$ (2012) The baseline and longitudinal changes of PCC connectivity in mild cognitive impairment: A combined structure and resting-state fMRI study. PLoS One 7, e36838.

[24] Serra L, Cercignani M, Mastropasqua C, Torso M, Spanò B, Makovac E, Viola V, Giulietti G, Marra C, Caltagirone C, Bozzali M, Galimberti D (2016) Longitudinal changes in functional brain connectivity predicts conversion to Alzheimer's disease. J Alzheimers Dis 51, 377-389.

[25] Lampit A, Hallock H, Suo C, Naismith SL, Valenzuela M (2015) Cognitive training-induced short-term functional and long-term structural plastic change is related to gains in global cognition in healthy older adults: A pilot study. Front Aging Neurosci 7, 14.

[26] Wells RE, Yeh GY, Kerr CE, Wolkin J, Davis RB, Tan Y, Spaeth R, Wall RB, Walsh J, Kaptchuk TJ, Press D, Phillips RS, Kong J (2013) Meditation's impact on default mode network and hippocampus in mild cognitive impairment: A pilot study. Neurosci Lett 556, 15-19.

[27] van Paasschen J, Clare L, Yuen KSL, Woods RT, Evans SJ, Parkinson CH, Rugg MD, Linden DEJ (2013) Cognitive rehabilitation changes memory-related brain activity in people with Alzheimer disease. Neurorehabil Neural Repair 27, 448-459.

[28] Förster S, Buschert VC, Teipel SJ, Friese U, Buchholz H-G, Drzezga A, Hampel H, Bartenstein P, Buerger K (2011) Effects of a 6-month cognitive intervention on brain metabolism in patients with amnestic MCI and mild Alzheimer's disease. J Alzheimers Dis 26(Suppl 3), 337348 . 
[29] McKhann GM, Knopman DS, Chertkow H, Hyman BT, Jack CR Jr, Kawas CH, Klunk WE, Koroshetz WJ, Manly JJ, Mayeux R, Mohs RC, Morris JC, Rossor MN, Scheltens P, Carrillo MC, Thies B, Weintraub S, Phelps $\mathrm{CH}$ (2011) The diagnosis of dementia due to Alzheimer's disease: Recommendations from the National Institute on Aging-Alzheimer's Association workgroups on diagnostic guidelines for Alzheimer's disease. Alzheimers Dement 7, 263-269.

[30] World Health Organization (1993) The ICD-10 classification of mental and behavioural disorders: Diagnostic criteria for research, Drugs used in Skin Diseases, World Health Organization, Geneva.

[31] Brueggen K, Kasper E, Ochmann S, Pfaff H, Webel S, Schneider W, Teipel S (2017) Cognitive rehabilitation in Alzheimer's disease: A controlled intervention trial. J Alzheimers Dis 57, 1315-1324.

[32] Kurz A, Thone-Otto A, Cramer B, Egert S, Frolich L, Gertz H-J, Kehl V, Wagenpfeil S, Werheid K (2012) CORDIAL: Cognitive rehabilitation and cognitive-behavioral treatment for early dementia in Alzheimer disease: A multicenter, randomized, controlled trial. Alzheimer Dis Assoc Disord 26, 246-253.

[33] Buschert VC, Friese U, Teipel SJ, Schneider P, Merensky W, Rujescu D, Moller H-J, Hampel H, Buerger K (2011) Effects of a newly developed cognitive intervention in amnestic mild cognitive impairment and mild Alzheimer's disease: A pilot study. J Alzheimers Dis 25, 679-694.

[34] Erzigkeit H, Lehfeld H, Peña-Casanova J, Bieber F, Yekrangi-Hartmann C, Rupp M, Rappard F, Arnold K, Hindmarch I (2001) The Bayer-Activities of Daily Living Scale (B-ADL): Results from a validation study in three European countries. Dement Geriatr Cogn Disord 12, 348358.

[35] Oswald WD, Fleischmann UM (1983) The Nuremberg Gerontopsychological Inventory as a psychometric assessment in aging brain. Aging 23, 121-129.

[36] Morris JC, Mohs RC, Rogers H, Fillenbaum G, Heyman A (1987) Consortium to establish a registry for Alzheimer's disease (CERAD) clinical and neuropsychological assessment of Alzheimer's disease. Psychopharmacol Bull 24, 641-652.

[37] Yesavage JA, Brink TL, Rose TL, Lum O, Huang V, Adey M, Leirer VO (1982) Development and validation of a geriatric depression screening scale: A preliminary report. J Psychiatr Res 17, 37-49.

[38] Smith SC, Lamping DL, Banerjee S, Harwood R, Foley B, Smith P, Cook JC, Murray J, Prince M, Levin E, Mann A, Knapp M (2005) Measurement of health-related quality of life for people with dementia: Development of a new instrument (DEMQOL) and an evaluation of current methodology. Health Technol Assess 9, 1-93, iii-iv.

[39] Gaser C, Volz HP, Kiebel S, Riehemann S, Sauer H (1999) Detecting structural changes in whole brain based on nonlinear deformations-application to schizophrenia research. Neuroimage 10, 107-113.

[40] Chao-Gan Y, Yu-Feng Z (2010) DPARSF: A MATLAB toolbox for "pipeline" data analysis of resting-state fMRI. Front Syst Neurosci 4, 13.

[41] Ashburner J (2007) A fast diffeomorphic image registration algorithm. Neuroimage 38, 95-113.

[42] Nowinski WL, Fang A, Nguyen BT, Raphel JK, Jagannathan L, Raghavan R, Bryan RN, Miller GA (1997) Multiple brain atlas database and atlas-based neuroimaging system. Comput Aided Surg 2, 42-66.
[43] Sestieri C, Corbetta M, Romani GL, Shulman GL (2011) Episodic memory retrieval, parietal cortex, and the default mode network: Functional and topographic analyses. $\mathrm{J} \mathrm{Neu}$ rosci 31, 4407-4420.

[44] Buckner RL, Andrews-Hanna JR, Schacter DL (2008) The brain's default network: Anatomy, function, and relevance to disease. Ann N Y Acad Sci 1124, 1-38.

[45] van Paasschen J, Clare L, Yuen KSL (2013) Cognitive rehabilitation changes memory-related brain activity in people with Alzheimer disease. Neurorehabil Neural Repair 27, 448-459.

[46] Habas C, Kamdar N, Nguyen D, Prater K, Beckmann CF, Menon V, Greicius MD (2009) Distinct cerebellar contributions to intrinsic connectivity networks. J Neurosci 29, 8586-8594.

[47] Castellazzi G, Palesi F, Casali S, Vitali P, Sinforiani E, Wheeler-Kingshott CAM, D'Angelo E (2014) A comprehensive assessment of resting state networks: Bidirectional modification of functional integrity in cerebro-cerebellar networks in dementia. Front Neurosci 8, 223.

[48] Bai F, Liao W, Watson DR, Shi Y, Yuan Y, Cohen AD, Xie C, Wang Y, Yue C, Teng Y, Di Wu, Jia J, Zhang Z (2011) Mapping the altered patterns of cerebellar resting-state function in longitudinal amnestic mild cognitive impairment patients. J Alzheimers Dis 23, 87-99.

[49] Guo CC, Tan R, Hodges JR, Hu X, Sami S, Hornberger M (2016) Network-selective vulnerability of the human cerebellum to Alzheimer's disease and frontotemporal dementia. Brain 139, 1527-1538.

[50] Thompson RF, Kim JJ (1996) Memory systems in the brain and localization of a memory. Proc Natl Acad Sci U S A 93, 13438-13444.

[51] De Zeeuw CI, Ten Brinke MM (2015) Motor learning and the cerebellum. Cold Spring Harb Perspect Biol 7, a021683.

[52] van Halteren-van Tilborg, Ilse ADA, Scherder EJA, Hulstijn W (2007) Motor-skill learning in Alzheimer's disease: A review with an eye to the clinical practice. Neuropsychol Rev 17, 203-212.

[53] Zhong Y, Huang L, Cai S, Zhang Y, Deneen KM, von, Ren A, Ren J (2014) Altered effective connectivity patterns of the default mode network in Alzheimer's disease: An fMRI study. Neurosci Lett 578, 171-175.

[54] Jones DT, Knopman DS, Gunter JL, Graff-Radford J, Vemuri P, Boeve BF, Petersen RC, Weiner MW (2016) Cascading network failure across the Alzheimer's disease spectrum. Brain 139, 547-562.

[55] Dyrba M, Barkhof F, Fellgiebel A, Filippi M, Hausner L, Hauenstein K, Kirste T, Teipel SJ (2015) Predicting prodromal Alzheimer's disease in subjects with mild cognitive impairment using machine learning classification of multimodal multicenter diffusion-tensor and magnetic resonance imaging data. J Neuroimaging 25, 738-747.

[56] Zhang H-Y, Wang S-J, Xing J, Liu B, Ma Z-L, Yang M, Zhang Z-J, Teng G-J (2009) Detection of PCC functional connectivity characteristics in resting-state fMRI in mild Alzheimer's disease. Behav Brain Res 197, 103-108.

[57] Yao H, Zhou B, Zhang Z, Wang P, Guo Y, Shang Y, Wang L, Zhang X, An N, Liu Y (2014) Longitudinal alteration of amygdalar functional connectivity in mild cognitive impairment subjects revealed by resting-state FMRI. Brain Connect 4, 361-370.

[58] Schulz KF, Altman DG, Moher D (2011) CONSORT 2010 statement: Updated guidelines for reporting parallel group randomised trials. Int J Surg 9, 672-677. 